\title{
ATMOSPHERES AND ABUNDANCES OF BLUE HORIZONTAL BRANCH STARS AND RELATED OBJECTS
}

\author{
U. HEBER \\ Institut für Theoretische Physik und \\ Sternwarte der Universität Kiel \\ Olshausenstr. 40, D-2300 Kiel 1 \\ Federal Republic of Germany
}

\begin{abstract}
Spectroscopic analyses of blue horizontal branch stars, subluminous B- and O-stars and PG 1159 stars are reviewed. These classes of stars trace stellar evolution from the horizontal branch towards the white dwarf cooling sequence. The impact of Non-LTE model atmospheres for the analyses of sdO and PG 1159 stars is outlined. The resulting atmospheric parameters and abundances are used to obtain constraints on the evolutionary status of the different classes of stars. The sdB stars form a homogeneous group and can be identified with models of the extended horizontal branch. Abundance anomalies (deficiency of helium and some metals, enrichment of ${ }^{3} \mathrm{He}$ ) observed in Horizontal Branch stars and sdB stars do not give hints to the stars' evolutionary history but are caused by atmospheric diffusion. The class of subluminous $\mathrm{O}$ stars is much less homogenous and two subclasses can be defined: the "compact" sdO stars probably evolved from the extended horizontal branch and are hence successors of the sdBs, whereas some sdOs of relatively low gravity are in a post-AGB stage of evolution. The third class, the PG 1159 stars, are amongst the hottest stars known with effective temperatures exceeding $100,000 \mathrm{~K}$ and are immediate progenitors of the white dwarfs. The abundance pattern is dominated by carbon and helium with a significant admixture of oxygen, whereas hydrogen and nitrogen are trace elements only. As in the case of the (helium rich) sdO stars these abundances indicate that nuclear processed material is exposed at the stellar surface. The origin of the PG 1159 stars as well as of the "low gravity" sdO stars can be explained by the born-again post-AGB star scenario of Iben et al. (1983).
\end{abstract}

\section{Introduction}

Some galactic globular clusters contain very blue horizontal branch stars, which appear to be separated from the main bulk of the horizontal branch by a gap in some cases. The classical example for such a HB morphology is NGC 6752 (cf. the colour-magnitude diagram by Cannon, 1981). Stars above the HB gap are considered to be normal horizontal branch stars of spectral type B (HBB stars), whereas the very blue objects below the gap may be termed extended horizontal branch (EHB) stars. Since these cluster EHB stars are faint, very little information about their atmospheric abundances is available. Hence it is worthwhile to look for brighter analogs amongst the field stars. Greenstein and Sargent (1974) already pointed out in their pioneering work that sdB and sdO stars might be identified with the extended horizontal branch. These stars belong to population I as indicated by their kinematical properties (Baschek and Norris, 1975) and scale height (Heber, 1986, Green and Liebert, 1987, Moehler et al., 1990).

This review will be restricted to recent analyses of HBB- and sdB-stars (section 2), sdO 
stars (section 3) and the recently discovered very hot pre white dwarfs of the PG 1159 class (section 4). This list of programme stars traces stellar evolution from the horizontal branch to the white dwarf cooling sequence and is a sequence in increasing temperature. Therefore, we shall undertake a journey through the HR-diagram starting from temperatures, typical for B-star, of about $15,000 \mathrm{~K}$ to extremely high values of $150,000 \mathrm{~K}$ or so.

Summaries of earlier work (not mentioned here) are given in previous reviews (e.g. Heber, 1987a, b; Kudritzki, 1987; Vauclair and Liebert, 1987).

\section{Horizontal branch B- and sdB-stars}

The atmospheres of HBB- and $\mathrm{sdB}^{*}$ stars are, in general, helium deficient. Exceptions have been reported by Heber et al. (1988), de Boer et al. (1988) and Viton et al. (1990). It has been realized that the helium deficiency in the HBB and sdB atmospheres is caused by atmospheric diffusion. Hence the observed abundance patterns are of little help in order to identify evolutionary links to other classes of stars. They do not reflect the star's evolutionary history but are the result of the competition between gravity and radiative acceleration in their present stage of evolution.

Spectroscopic analyses of HB stars have been carried out only for a few galactic globular clusters, out of which NGC 6752 has been studied most extensively (Heber et al., 1986; Glaspey et al., 1989). Recently, Crocker et al. (1989) derived atmospheric parameters for HB stars in M 3, M 5, M 15, M 92 and NGC 288. Their observed positions in the $\left(T_{\text {eff }}\right.$, $\log g$ ) plane fit theoretical predictions well only in the case of M 5. In other clusters several HB stars were found to lie at lower gravities than predicted by standard evolution theory. Helium and iron abundances for NGC 6752 stars agree with predictions of diffusion theory.

Much more information is available for field stars. In Fig. 1 atmospheric parameters for 75 stars (taken mainly from sources listed by Heber 1987a, and from Moehler et al., 1990, Viton et al., 1990) are summarized. Effective temperatures are derived from ultraviolet fluxes, Strömgren photometry or the He I/He II ionization equilibrium. Gravities have been determined from Balmer line profiles. NLTE effects are important only for the hottest stars in this sample (Hunger et al., 1981, Heber et al., 1984), whereas line blanketed LTE models are sufficient below $35000 \mathrm{~K}$ at the moderately high gravities in question $(5.0<\log g<6.0)$.

Constraints on the evolutionary status of the HBB and sdB stars can be gained from their atmospheric parameters by comparing them to predictions of evolutionary calculations (see Fig. 1). Before we draw conclusions from such a comparison it is necessary to explain why we have to distinguish the extended HB from the normal HB. The internal structure of an extended horizontal branch star namely differs markedly from that of a HB star. The luminosity of the HB star's hydrogen burning shell equals or even exceeds the luminosity of the helium burning core whereas in an EHB star the luminosity of the hydrogen burning shell is negligibly small. Hence the internal structure of an EHB star bears great resemblence to a helium main sequence star of half a solar mass and its further evolution should proceed similar to that of the latter. Recent calculations by Caloi (1989) show that this is indeed the case. The EHB models evolve bluewards (see Fig. 1) directly

*Baschek and Norris (1975) introduced the term sdOB in order to classify hot subluminous stars with spectra intermediate between sdB and sdO stars. Since the atmospheric properties of the sdOBs are so similar to that of the sdBs, we shall subsume the sdOBs under the term sdB throughout this paper. 
to the white dwarf cooling sequence thereby omitting the second red giant branch. For this reason it is justified to distinguish EHB models from HB models.

From Fig. 1 it is evident that the observed positions of the sdB stars lie close to predictions for the EHB. All stars lie on or above the zero age EHB. Part of the observed width of the $\mathrm{sdB}$ distribution may be explained by evolution. However, the observed distribution appears to extend to lower gravities than predicted by Caloi's evolutionary tracks - a phenomenon that is also observed in several globular cluster HBs (Crocker et al., 1989).

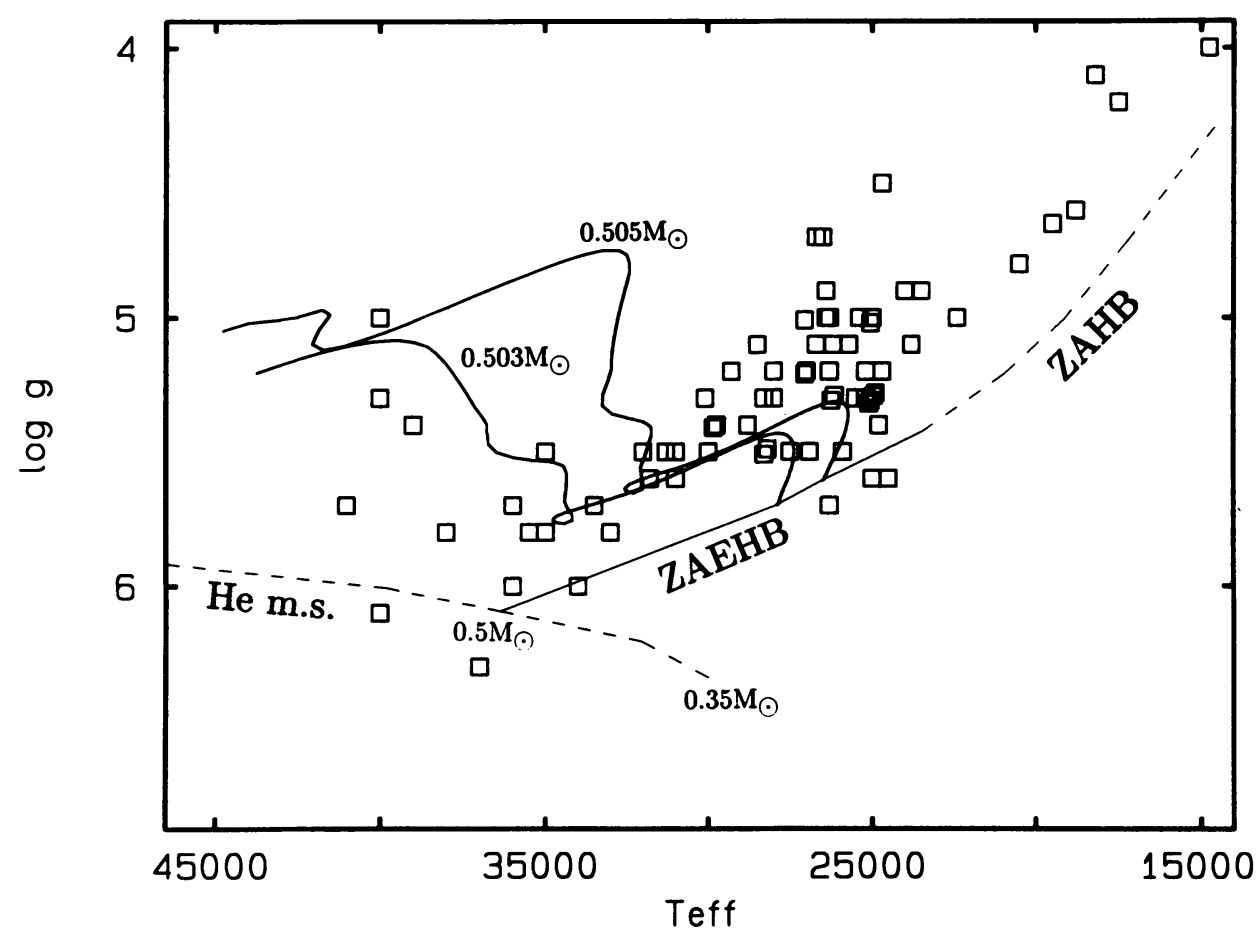

Figure 1: Comparison of the observed position of $\mathrm{sdB}$ stars to evolutionary models. The zero age horizontal branch (ZAHB), the zero age extended horizontal branch (ZAEHB), the helium main sequence (He-m.s.) and two evolutionary tracks for masses of $0.503 M_{\odot}$ and $0.505 M_{\odot}(\mathrm{Caloi}, 1989)$ are shown.

Helium is deficient in the vast majority of the programme stars by factors ranging from 2 to 300 . Trends for the helium abundance with $T_{\text {eff }}$ or $\log g$ cannot be established. Although the helium deficiencies are undoubtedly due to diffusion, parameterfree diffusion models cannot reproduce the observed abundances at least for the sdOB subclass (Michaud et al., 1989).

Besides the helium deficiency other anomalies with respect to isotopic abundance ratios and metal abundances are to be expected because of differences in their respective atomic weights. One such isotopic ratio which is easily accessible to high resolution spectroscopy 
is the ${ }^{3} \mathrm{He} /{ }^{4} \mathrm{He}$ ratio. ${ }^{3} \mathrm{He}$ is not detectable in the spectra of normal stars because of its presumedly low abundance (solar abundance: ${ }^{3} \mathrm{He} / \mathrm{H}=10^{-5}$ ). However, a few peculiar main sequence stars are known to show ${ }^{3} \mathrm{He}$, the protoype being $3 \mathrm{Cen} \mathrm{A}$ (Sargent and Jugaku, 1961). Feige 86 was the first HBB star found to show evidence for the presence of ${ }^{3} \mathrm{He}$ (Hartoog, 1979). Recently a systematic search for ${ }^{3} \mathrm{He}$ in the spectra of $14 \mathrm{HBB}$ and sdB stars using high resolution ESO-CASPEC spectra was carried out (Heber, 1990, see also Heber, 1987a). These spectra allowed precise wavelengths of two He I lines $(6678 \AA$, $5875 \AA$ ) as well as radial velocities (from hydrogen and metal lines) to be measured. He I, $6678 \AA$ exhibits the largest isotopic line shift $(0.5 \AA)$ among the He I lines in the visual spectral range, whereas He I, $5875 \AA$ provides a useful check since its isotopic line shift is negligibly small $(0.04 \AA)$. Indeed, remarkably large shifts of the $6678 \AA$ line were measured for three stars $(0.43 \AA$ for SB 290 and PHL $25 ; 0.45 \AA$ for PHL 382) as can be seen from Fig. 2. These line shifts are larger than found previously in any ${ }^{3} \mathrm{He}$ star and indicate that ${ }^{4} \mathrm{He}$ is almost entirely replaced by ${ }^{3} \mathrm{He}$ in these three stars.
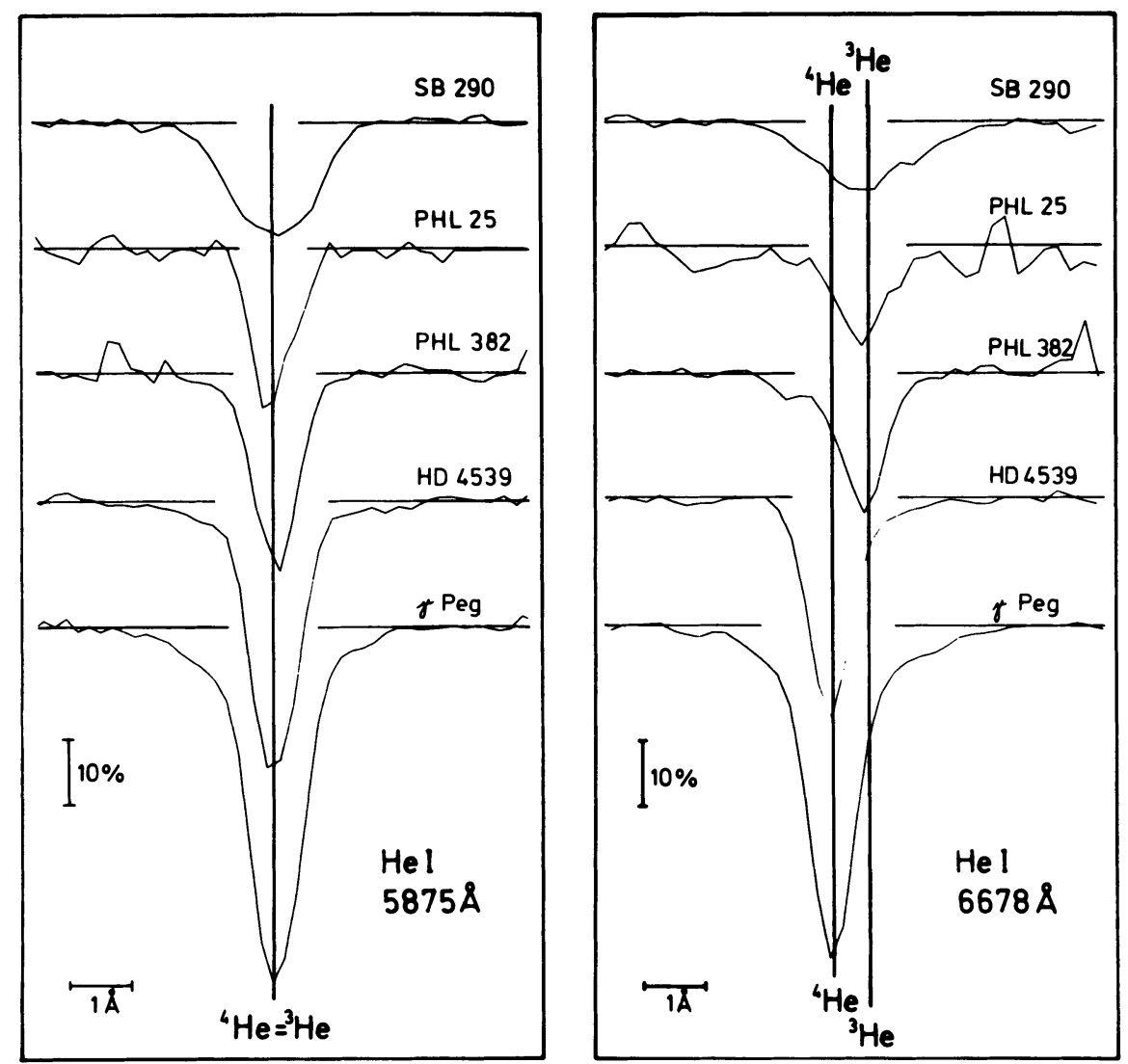

Figure 2: He I, $5875 \AA$ (left panel) and He I, $6678 \AA$ (right panel) from ESO-CASPEC spectra of 4 subluminous $B$ stars and $\gamma$ Peg. Line positions for pure ${ }^{3} \mathrm{He}$ and pure ${ }^{4} \mathrm{He}$ are indicated. Note the line shift of He I, 6678 in SB 290, PHL 25 and PHL 382. 
Let us now turn to the metal abundance pattern. Most of what is known today resulted from high resolution IUE studies. The analyses mainly aimed at the nucleogenetically important elements carbon and nitrogen and at silicon. The results for 11 stars are summarized by Lamontagne et al. (1985). The helium deficiency is accompanied by large deficiencies of carbon and silicon whereas nitrogen is almost normal (see Fig. 6 of Lamontagne etal., 1985). There appears to be a trend for the $\mathrm{C}$ and $\mathrm{Si}$ abundance to decrease with inceasing $T_{\text {eff. }}$. These observed $\mathrm{C}, \mathrm{N}$ and $\mathrm{Si}$ abundance can be reproduced by diffusion models if mass loss at a small rate (of the order of the solar one, i.e. $10^{-14} M_{\odot} / y r$ ) is considered (Michaud et al., 1985).

However, the diffusion process does not necessarily lead to a depletion of all the metals. An overabundance of phosphorus in the HBB star Feige 86 for example has already been noted by Baschek and Sargent (1976). Recently, high resolution blue optical spectra obtained with ESO-CASPEC revealed the existence of another abundance anomaly (Heber, 1990). The HBB stars PHL 25 and PHL 1434 showed an enrichment of chlorine by factors of 20 to 40 with respect to the sun. The chlorine anomaly is illustrated in Fig. 3, where the spectra of PHL 25 and PHL 1434 are compared to the normal B star $\gamma$ Peg. The chlorine and phosphorus enrichment is probably caused by the radiative acceleration being large for these elements ("levitation").

The observed abundance pattern of $\mathrm{HBB}$ and sdB stars are still a challenge to theoreticians in the field of diffusion processes.

Figure 3:

Portion of the CASPEC spectra of PHL 25 and PHL 1434 compared to $\gamma$ Peg. Note the strength of the $\mathrm{Cl}$ II lines in PHL 25 and PHL 1434.

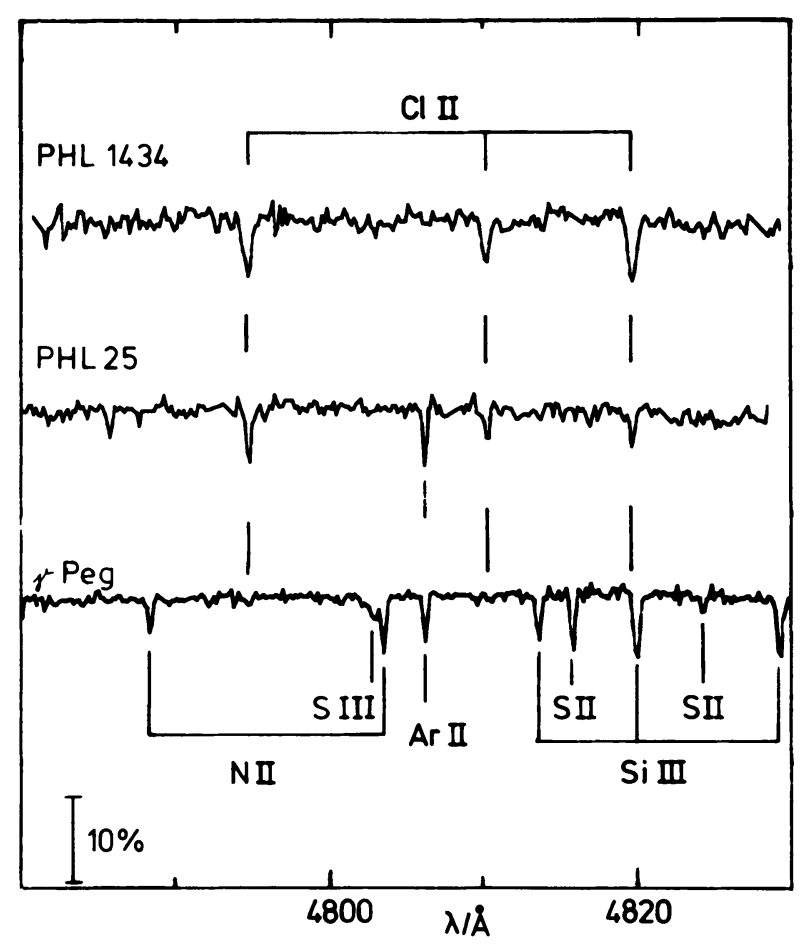




\section{Subluminous O-stars}

We now turn to the subluminous $O$ stars which are hotter $\left(T_{\text {eff }}>35000 \mathrm{~K}\right)$ than the sdBs and have, in general, helium rich atmospheres (exceptions are reported by Schönberner and Drilling, 1984) in contrast to the sdB and HBB stars discussed in section 2. Because of the large effective temperatures in question, NLTE effects become very important. Two characteristic effects become apparent (see e.g. Fig. 2 of Werner et al., 1990): the NLTE profile is considerably stronger than the LTE one and occassionally displays a central emission reversal.

Up to recently, only very few NLTE analyses of sdO stars have been performed (e.g. Hunger et al., 1981). The reason for this is that problems with the classical NLTE technique, the complete linearisation method of Auer and Mihalas (1969), arose. Hardly any heliumrich model could be converged in a parameter range relevant to the sdO stars (see Husfeld et al., 1989). Moreover, the complete linearisation technique allowed only a very limited number of atomic levels to be treated in NLTE. Both drawbacks have now been overcome by a new method to construct NLTE model atmospheres, the socalled Accelerated Lambda Iteration developed by Werner and Husfeld $(1985)$ and Werner $(1986,1988)$. This technique has been applied by Dreizler et al. (1990) and Rauch et al. (1990) to analyse sdO stars. Up to now, atmospheric parameters for 32 subluminous 0 stars have been derived and are summarized in Fig. 4. Two groups of sdO stars can be distinguished with respect to their hydrogen content: stars marked as squares do not show any trace of hydrogen whereas the triangles denote sdOs with hydrogen and helium composed atmospheres $(\mathrm{H} / \mathrm{He}=1$ by number, typically) The class of sdO stars is far less homogenous than the sdB class with respect to their atmospheric parameters since they are spread out all over the $\left(T_{\text {eff }}\right.$, $\log g$ )-plane. In Fig. 4, evolutionary tracks starting from the EHB as well as those starting from the asymptotic giant branch (AGB) are also displayed. As can be seen many of the sdO stars lie close to the post-EHB tracks and therefore might have evolved from the sdB stars. However, several sdOs (of relatively low gravities) cannot be post-EHB stars but are identified as descendants of the AGB stars because they lie close to the post-AGB tracks in Fig. 4. These stars are found in the same region of the $\left(T_{\text {eff }}, \log g\right)$-plane as the Central stars of Planetary Nebulae (CSPN). Hence the sdO stars form two distinct groups with respect to their evolutionary history: The "compact" sdOs evolving from the EHB and the "low gravity" sdOs being post-AGB objects similar to the CSPNs.

For the "low gravity" sdOs the question then arises why no planetary nebulae are visible around them. A clue to this question may come from the atmospheric abundances of carbon and nitrogen. Husfeld et al. (1989) and Rauch et al. (1990) carried out NLTE analyses of four sdO stars belonging to the "low gravity" subclass. The problems inherent in these analyses may be exemplified in the case of KS 292 (Rauch et al., 1990). The most important spectral lines of carbon and nitrogen are due to $\mathrm{C} \mathrm{IV}$ and $\mathrm{N} \mathrm{V}$ and arise mainly from highly excited levels ( $n=5$ or higher). This fact renders their analysis difficult for two reasons: i) Very elaborate model atoms are required in NLTE line formation calculations in order to obtain reliable occupation numbers for these high lying levels. Rauch et al. (1990), for example, had to treat 48 NLTE levels and 146 line transitions of C IV in the statistical equilibrium equations. ii) The highly excited levels are close to degeneracy rendering the treatment of the line broadening difficult. The broadening mechanism ranges between the linear and the quadratic Stark effect. A gradual change from quadratic to linear stark effect occurs with increasing principal quantum number. Unfortunately, an exact theory for this 
intermediate case is not available. An approximate treatment has been proposed by Werner et al. (1990) and Rauch et al. (1990) who determined correction factors to Holtsmark's statistical theory of pressure broadening by comparison with laboratory experiments. (For details see Werner et al., 1990).

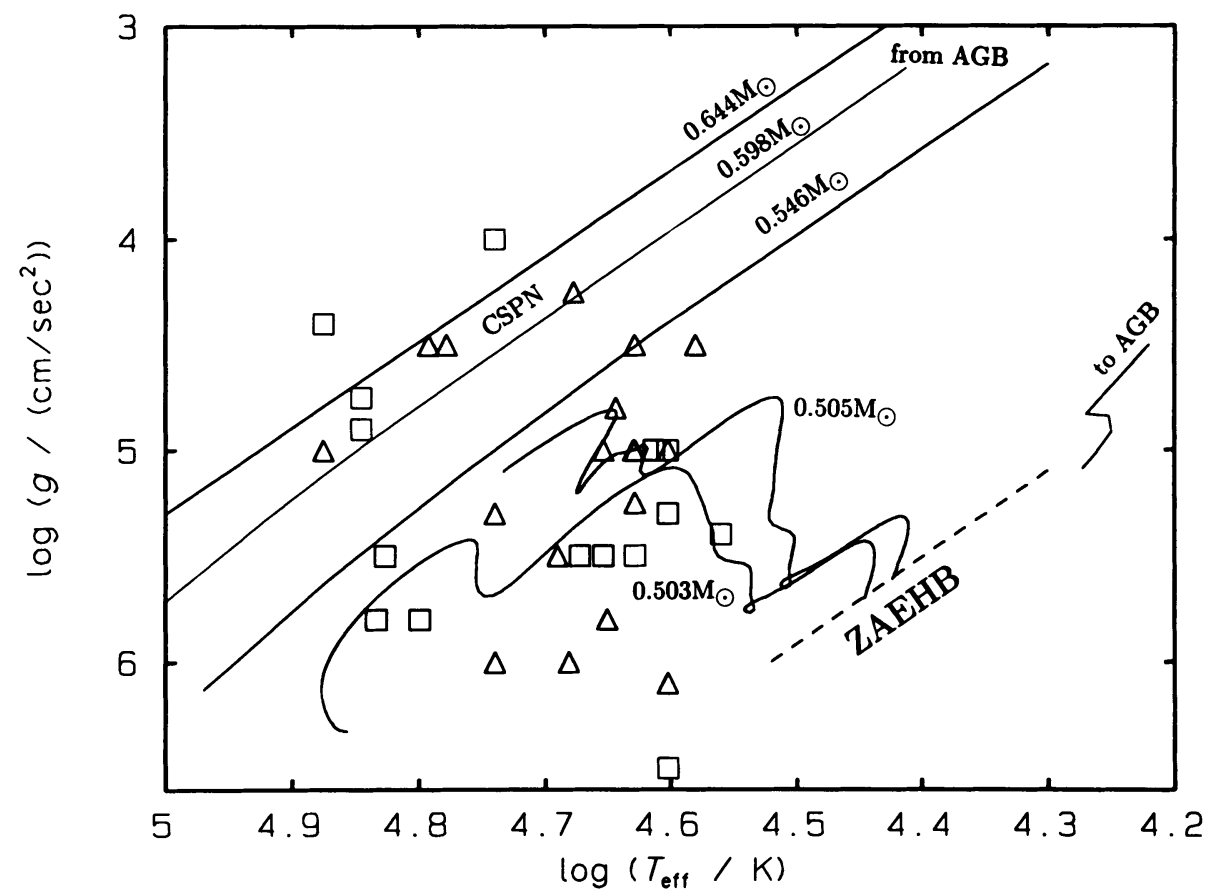

Figure 4: Position of the sdO stars in the $\left(T_{\text {eff }}, \log g\right)$-plane and comparison with evolutionary tracks starting from the AGB (Schönberner, 1979, 1983) and starting from the EHB (Caloi, 1989). Squares: stars with no hydrogen detected; Triangles: Hydrogen present.

The results of the abundance analyses for $\mathrm{C}$ and $\mathrm{N}$ can be summarized as follows. Nitrogen is overabundant in all four stars (by factors between 8 and 30) and carbon is also enriched in LSE 259, LSE 159 and KS 292 (factors 5 to 15) whereas LSE 263 is carbon deficient by a factor of about ten. These abundance patterns indicate that we see material at the stellar surface that has been processed by the $\mathrm{CN}$ cycle ( $\mathrm{N}$ enrichment, $\mathrm{C}$ deficiency) as well as the products of the $3 \alpha$-process (C enrichment, not for LSE 263). In KS 292 there is also a large fraction of unprocessed material as indicated by the presence of hydrogen.

How can these peculiar abundance pattern as well as the stars' position in the HRD be explained in the context of stellar evolution? Various evolutionary scenarios are conceivable (see Husfeld et al., 1989 and Rauch et al., 1990 for a detailed discussion). Heber and Hunger (1987) suggested that the "born-again post-AGB-star" scenario of Iben et al. (1983) is the most promising one. According to this scenario a post-AGB star suffers a late helium shell flash bringing it back onto the AGB for a second time. Most of its hydrogen envelope reduced to $10^{-4} M_{\odot}$ during the first descend from the AGB can now be removed if there 
is a second superwind phase. During the second descend a modest mass loss $\left(10^{-8} M_{\odot} / y r\right.$ is sufficient to strip off the remaining hydrogen layer. The star is now powered by helium burning for which evolutionary time scales are so long that a planetary nebula, which has possibly been ejected during the first departure from the AGB, has long been dispersed. This explains why no nebulosities could be detected.

In summary, we can identify two evolutionary channels leading to the sdO stage: one starting from the extended horizontal branch and explaining the compact sdO and another one starting from the asymptotic giant branch and leading to the "low gravity" sdOs possibly via a second loop in the HRD caused by a final helium shell flash.

\section{PG 1159 stars}

Before the white dwarf cooling sequence is reached we find another class of extremely hot pre white dwarf stars which have only recently been discovered and are named after the prototype PG 1159-035 (Mc Graw et al., 1979). Like many sdO stars, the PG 1159 stars are devoid of hydrogen. Spectroscopically they are distinguished from sdOs and hydrogen deficient degenerates (DO) by a characteristic absorption trough shortwards of $4700 \AA$ caused by He II, $4686 \AA$ and many C IV lines. The PG 1159 stars have received great attention since PG 1159-035 and six other members of the class have been discovered to exhibit low amplitude multiperiodic light variations with periods between 5 min. and 30 min. and hence define a new class of variable stars (the GW Vir stars). These variations have been identified as non-radial g-mode pulsations. The GW Vir stars are amongst the first stars, to which the techniques of helioseismology can be applied. Hence they open up the field of "asteroseismology" which allows interior layers far below the photosphere to be probed and information about the basic stellar parameters such as the mass, rotation period and magnetic field strength to be derived.

A prerequisite for the asteroseismological analysis is the precise knowledge of the photospheric parameters and abundances. In particular the element abundances are important to identify the pulsation driving mechanism. However, no reliable spectral analysis could be performed because NLTE model atmospheres for very high effective temperatures and "exotic" chemical composition were lacking. The NLTE modelling turned out to be even more complicated than in the case of the sdO stars (section 3) because $\mathrm{C}$ and $\mathrm{O}$ are so abundant that their opacities strongly influence the atmospheric structure and detailed $\mathrm{C}$ and $\mathrm{O}$ model atoms (besides $\mathrm{He}$ ) need to be included in the NLTE model atmosphere calculations. The construction of such elaborate models has become possible only by the developement of the "Accelerated Lambda Iteration" technique, which was applied recently by Werner et al. (1990) to analyse optical spectra of four PG 1159 stars. A profile fit for the trough region of PG 1159-035 is shown in Fig. 5. The results confirmed that the PG 1159 stars are amongst the hottest stars know with effective temperatures ranging from $100,000 \mathrm{~K}$ to $140,000 \mathrm{~K}$. Their temperatures and gravities $(\log g=7)$ are higher than those of the sdO stars indicating that the PG 1159 stars are further evolved than the sdO stars. Even more exciting are the resulting abundances: Carbon ( $50 \%$ by mass) and helium (33\%) are dominating the abundance pattern and oxygen is also present in a significant amount $(17 \%)$, whereas nitrogen is a trace element only $(<0.4 \%)$. These abundances are uniform within the four stars studied sofar and indicate that the non-radial pulsations are driven by cyclic ionisation of carbon and oxygen (Starrfield, 1987). However, 
only two of the four PG 1159 stars analysed by Werner et al. (1990) are pulsating. The variable star PG $1707+427$ and the non-variable PG $1424+535$ have identical atmospheric parameters and abundances (to within observational limits). The same holds for the pair PG 1159-035 (variable) and PG 1520+525 (non-variable). Hence, the physical parameter which determines whether a PG 1159 star pulsates or not, still remain obscure.

As in the case of the sdO stars (section 3 ) the surface abundance pattern is a clue to the stars' evolutionary history. The atmospheric abundances resemble closely those found in the helium buffer layers of post-AGB star models (Iben, 1984). However, a considerable amount of matter has to be removed from the star before these deep layers become visible at the stellar surface. As pointed out by Werner et al. (1990) this can only be achieved by the born-again post-AGB star scenario of Iben et al. (1983). Calculated surface abundances (Iben, 1984) are in quantative agreement with observations.

Since the born-again post-AGB star scenario can explain the "low gravity" sdOs as well as the PG 1159 stars, one might speculate whether there could be an evolutionary link between these two groups of stars. Since the abundances of carbon and oxygen are much higher in the PG 1159 stars than in the sdOs additional mass loss is required to remove helium rich material and to transform a sdO star into a PG 1159 star. Mass loss rates need to be determined for the sdO stars in order to check this speculation. Another evolutionary link of the PG 1159 stars to the Central Stars of Planetary Nebulae of spectral type WC has been proposed by Werner et al. (1990) since their abundances appear to be very similar to those of the PG 1159 stars.

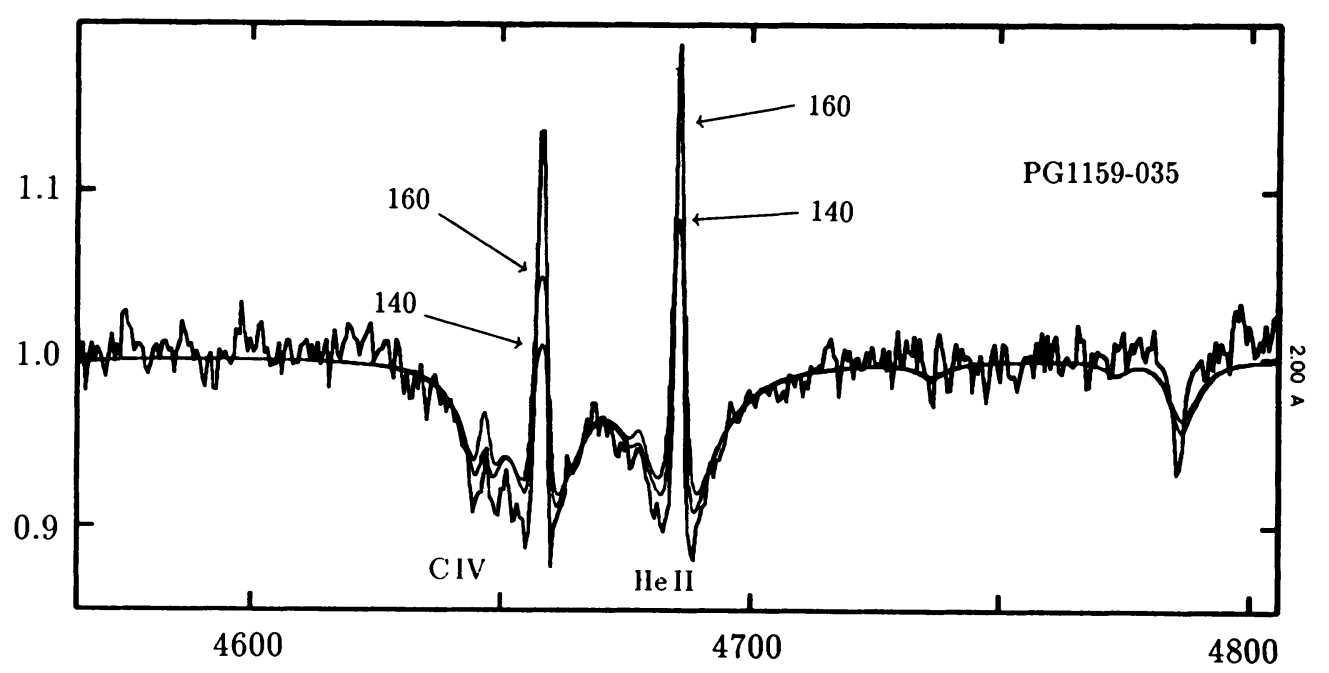

Figure 5: Comparison of the observed spectrum (with the characteristic absorption trough) of PG 1159-035 to theoretical NLTE profiles for $T_{\text {eff }}=120,000 \mathrm{~K}, 140,000 \mathrm{~K}$ and $160,000 \mathrm{~K}$ (from Werner et al., 1990). Note that the observed central emission reversals of C IV and He II can well be reproduced by static NLTE model atmospheres. 


\section{Future Work}

In the previous sections we have drawn a mosaic of stellar evolution from the horizontal branch to the white dwarf stage. Many questions concerning this mosaic need still to be answered. Only two of them shall be raised here.

i) What is the origin of the extended horizontal branch stars, i.e. the subluminous B-stars? As pointed out in the introduction the sdBs belong to the old disk population and their progenitor masses on the main sequence are between one and two solar masses. However, according to canonical theory of stellar evolution the extreme horizontal branch will be reached only by stars of very low mass. Hence the progenitors of the sdBs have to loose more mass in the course of evolution than predicted by theory. Since only $2 \%$ of the white dwarfs evolve from the extended horizontal branch (Heber, 1986), only $2 \%$ of the HB stars need to experience large mass loss. Such an extra mass loss might be triggered by the core helium flash at the tip of the first giant branch. An alternative scenario to explain the sdB stars has been developed by Iben and Tutukov (1985) and Iben (1990): A sdB star can be formed by merging two helium white dwarfs in a very close binary system. This scenario predicts a broader range of masses for the sdBs than the former scenario does. Hence, a decision between these scenarios might become possible when the mass distribution of sdBs could be established.

ii) According to our picture of post-EHB evolution many helium rich sdO stars evolve from the helium poor sdB stars (see section 3). How can a helium poor atmosphere be transformed into a helium rich one? Atmospheric convection can be excluded according to the calculations of Groth et al. (1985). More likely appears to be mixing induced by helium or hydrogen shell flashes (Caloi, 1989, 1990; Iben and Tutukov, 1985) or relaxation oscillations of a helium-burning star (Iben et al., 1986) which occur during the post-EHB evolution. Additional evolutionary calculations are required in order to help understanding the $\mathrm{sdB} / \mathrm{sdO}$ transition.

Finally, the hot horizontal branch stars and related objects discussed here might be responsible for the relatively strong ultraviolet flux emitted by elliptical galaxies (Greggio and Renzini, 1990). Future telescopes and instrumentations might be able to obtain spectra of resolvable stellar systems such as M 32 .

ACKNOWLEDGEMENTS. My thanks go to my colleagues in Kiel Stefan Dreizler, Kurt Hunger, Thomas Rauch and Klaus Werner. Many of the results reported here have been obtained with their collaboration. I also thank Professor Iben for fruitful discussion on evolutionary aspects. Travel grants by the DFG and the IAU are gratefully acknowledged.

\section{REFERENCES}

Auer, L.H., Mihalas, D. 1969: Astrophys. J. 158, 641.

Baschek, B., Norris, J. 1975: Astrophys. J. 199, 694.

Baschek, B., Sargent, A.I. 1976: Astron. Astrophys. 53, 47.

de Boer, K.S., Heber, U., Richtler, T. 1988: Astron. Astrophys. 202, 113.

Caloi, V. 1989: Astron. Astrophys. 221, 27.

Caloi, V. 1990: Astron. Astrophys. 232, 67.

Cannon, R.D. 1981: Proceedings of IAU Symp. No. 105: Observational tests of the stellar evolution theory, eds. A. Maeder and A. Renzini,Dordrecht: Reidel, p. 123.

Crocker, D.A., Rood, R.T., O'Connell, R.W. 1989: Astrophys. J. 332, 236.

Dreizler, S., Heber, U., Werner, K., Moehler, S., de Boer, K.S. 1990: Astron. Astrophys. 235, 234.

Glaspey, J.W., Michaud, G., Moffat, A.F.J., Demers, S. 1989: Astrophys. J. 339, 926. 
Green, R.F., Liebert, J. 1987: Proceedings of IAU colloquium No. 95: The Second Conference on Faint Blue Stars, eds. A.G.D. Philip, D.S. Hayes and J. Liebert, Schenectady: Davis Press , p. 261.

Greenstein, J.L., Sargent, A.I. 1974: Astrophys. J. Suppl. 28, 157.

Greggio, L., Renzini, A. 1990: ESO-preprint 697, 1.

Groth, H.G., Kudritzki, R.P., Heber, U. 1985: Astron. Astrophys. 152, 107.

Hartoog, M.R. 1979: Astrophys. J. 231, 161.

Heber, U. 1986: Astron. Astrophys. 155, 33.

Heber, U. 1987a: Mitt. astron. Ges. 70, 71.

Heber, U. 1987b: Proceedings of IAU colloquium No. 95: The Second Conference on Faint Blue Stars, eds. A.G.D. Philip, D.S. Hayes and J. Liebert, Schenectady: Davis Press , p. 79.

Heber, U. 1990: Astron. Astrophys., in preparation.

Heber, U., Hunger, K. 1987: Proceedings of IAU colloquium No. 95: The Second Conference on Faint Blue Stars, eds. A.G.D. Philip, D.S. Hayes and J. Liebert, Schenectady: Davis Press , p. 599.

Heber, U., Hamann, W.-R., Hunger, K., Kudritzki, R.P., Simon, K.P., Mendez, R.H. 1984: Astron. Astrophys. 136, 331.

Heber, U., Kudritzki, R.P., Caloi, V., Castellani, V., Danziger, J., Gilmozzi, R. 1986: Astron. Astrophys. 162, 171.

Heber, U., Dreizler, S., de Boer, K.S., Moehler, S., Richtler, T. 1988: Astron. Ges. Abs. Ser.1, 16.

Hunger, K., Gruschinske, J., Kudritzki, R.P., Simon, K.P. 1981: Astron. Astrophys. 95, 204.

Husfeld, D., Butler, K., Heber, U., Drilling, J.S. 1989: Astron. Astrophys. 222, 150.

Iben, I., Jr. 1984: Astrophys. J. 277, 333.

Iben, I., Jr. 1990: Astrophys. J. 353, 215.

Iben, I., Jr., Kaler, J.B., Truran, J.W., Renzini, A. 1983: Astrophys. J. 264, 605.

Iben, I., Jr., Tutukov, A.V. 1985: Astrophys. J. Suppl. 58, 661.

Iben, I., Jr., Fujimoto, M.Y., Sugimoto, D., Miyaji, S. 1986: Astrophys. J. 304, 217.

Kudritzki, R.P. 1987: Proceedings of IAU colloquium No. 95: The Second Conference on Faint Blue Stars, eds. A.G.D. Philip, D.S. Hayes and J. Liebert, Schenectady: Davis Press , p. 177.

Lamontagne, R., Wesemael, F., Fontaine, G., Sion, E.M. 1985: Astrophys. J. 299, 496.

Mc Graw, J.T., Starrfield, S., Liebert, J., Green, R.F. 1989: Proc. IAU coll. No. 53: White Dwarfs and Variable Degenerate Stars, eds. H.M. van Horn, V. Weidemann, Rochester, p. 377.

Michaud, G., Bergeron, P., Wesemael, F., Fontaine,G. 1985: Astrophys. J. 299, 741.

Michaud, G., Bergeron, P., Heber, U., Wesemael, F. 1989: Astrophys. J. 338, 417.

Moehler, S., Heber, U., de Boer, K.S. 1990: Astron. Astrophys. , in press.

Rauch, T., Heber, U., Hunger, K., Werner, K., Neckel, Th. 1990: Astron. Astrophys. , in press.

Sargent, W.L.W, Jugaku, J. 1961: Astrophys. J. 134, 777.

Schönberner, D. 1979: Astron. Astrophys. 79, 108.

Schönberner, D. 1983: Astrophys. J. 272, 708.

Schönberner, D., Drilling, J.S. 1984: Astrophys. J. 278, 702.

Starrfield, S. 1987: Proceedings of IAU colloquium No. 95: The Second Conference on Faint Blue Stars, eds. A.G.D. Philip, D.S. Hayes and J. Liebert, Schenectady: Davis Press, p. 309.

Vauclair, G., Liebert, J. 1987: in: Exploring the Universe with the IUE Satellite ed. Y. Kondo, Reidel, Dordrecht,' 355.

Viton, M., Deleuil, M., Tobin, W., Prevot, L., Bouchet, P. 1990: Astron. Astrophys. , in press.

Werner, K. 1986: Astron. Astrophys. 161, 177.

Werner, K. 1988: Astron. Astrophys. 204, 159.

Werner, K., Husfeld, D. 1985: Astron. Astrophys. 148, 417.

Werner, K., Heber, U., Hunger, K. 1990: Astron. Astrophys. , submitted. 